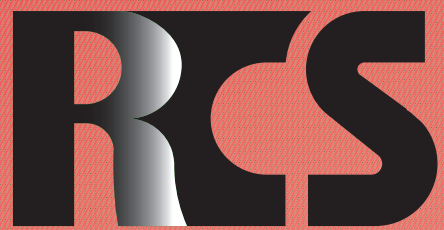

Depósito legal ppi $201502 Z U 4662$

Esta publicación científica en formato digital es continuidad de la revista impresa Depósito Legal: pp $197402 Z U 789$

- ISSN: 1315-9518 • ISSN-E: 2477-9431

Revista de Ciencias Sociales

Universidad del Zulia. Revista de la Facultad de Ciencias Económicas y Sociales Vol. XXVII. No. 2

Abril-Junio 2021

Esta publicación científica en formato digital es continuidad de la revista impresa Depósito Legal: pp $197402 Z$ Z789 ISSN: 1315-9518 


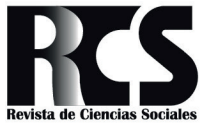

Revista de Ciencias Sociales (RCS). FCES - LUZ

Vol. XXVII, No. 2, 2021, pp. 467-483 Abril-Junio

- ISSN: 1315-9518 IISSN-E: 2477-9431
Como citar APA: Berrios-Riquelme, J., Ventura-León, J., Maluenda-Albornoz, J., y Barboza-Palomino, M. (2021). Propiedades psicométricas de la escala de amenaza grupal internalizada por inmigrantes latinoamericanos en Chile. Revista de Ciencias Sociales (Ve), XXVII(2), 467-483.

\title{
Propiedades psicométricas de la escala de amenaza grupal internalizada por inmigrantes latinoamericanos en Chile*
}

\author{
Berrios-Riquelme, José** \\ Ventura-León, Joséét*** \\ Maluenda-Albornoz, Jorge ${ }^{* * * *}$ \\ Barboza-Palomino, Miguel ${ }^{* * * * *}$
}

\section{Resumen}

La Escala de Amenaza Grupal Internalizada, es un instrumento que mide el prejuicio percibido por inmigrantes latinoamericanos desde la teoría de la amenaza. El objetivo de la investigación fue evaluar las propiedades psicométricas de esta escala en inmigrantes latinoamericanos que viven en Chile. A través de una metodología no-experimental, que tuvo un muestro no probabilístico, se realizaron dos estudios de corte transversal, en donde participaron un total de 722 inmigrantes. Los hallazgos del primer estudio, a partir de un análisis factorial exploratorio, evidencian una estructura unifactorial de la Escala de Amenaza Grupal Internalizada y un coeficiente omega de .87. Por su parte, los resultados del segundo estudio, a partir de un análisis factorial confirmatorio, corroboran la estructura factorial de la escala para un modelo de 7 ítems con adecuados índices de ajuste y cargas factoriales mayores a .50. Así también, se encontraron correlaciones estadísticamente significativas, inversas y moderadas con la Escala de Autoestima y la Escala de Identidad Étnica Multigrupo. Finalmente, se halló un coeficiente omega de .89. Se concluye, que la Escala de Amenaza Grupal Internalizada cuenta con adecuadas propiedades psicométricas para su uso con inmigrantes latinoamericanos que residen en Chile.

Palabras clave: Propiedades psicométricas; teoría de la amenaza; prejuicio percibido; inmigrantes latinoamericanos; Chile.

La presente investigación fue financiada por el proyecto UTA-MAYOR 6716/17.

** Doctor en Movilidad Humana. Profesor Asistente del Departamento de Ciencias Sociales de la Universidad de Tarapacá, Chile. E-mail: jberrios@uta.cl iD ORCID: https://orcid.org/0000-0003-2947-4739

*** Doctor en Psicología. Profesor de la Facultad de Ciencias de la Salud en la Universidad Privada del Norte, Perú. E-mail: jose.ventura@upn.pe (iD) ORCID: https://orcid.org/0000-0003-2996-4244

***** Doctor(c) en Psicología. Psicólogo. Becario del Departamento de Psicología de la Universidad de Concepción, Chile. E-mail: jorgemaluendaa@gmail.com iD ORCID: https://orcid.org/0000-0001-8148-4948

****** Magíster en Psicología con mención en Psicología Educativa. Docente Investigador de la Universidad Privada del Norte, Perú. E-mail: mbarbozapalomino@outlook.com.pe iD ORCID: https://orcid.org/0000-0001-8045-5491 


\title{
Psychometric properties of the group threat scale internalized by Latin American immigrants in Chile
}

\begin{abstract}
The Internalized Group Threat Scale is an instrument that measures the prejudice perceived by Latin American immigrants from the threat theory. The objective of the research was to evaluate the psychometric properties of this scale in Latin American immigrants living in Chile. Through a non-experimental methodology, which had a non-probabilistic sample, two cross-sectional studies were carried out, in which a total of 722 immigrants participated. The findings of the first study, based on an exploratory factor analysis, show a unifactorial structure of the Internalized Group Threat Scale and an omega coefficient of .87. On the other hand, the results of the second study, based on a confirmatory factor analysis, corroborate the factorial structure of the scale for a 7-item model with adequate fit indices and factor loads greater than .50. Likewise, statistically significant, inverse and moderate correlations were found with the Self-Esteem Scale and the Multigroup Ethnic Identity Scale. Finally, an omega coefficient of .89 was found. It is concluded that the Internalized Group Threat Scale has adequate psychometric properties for its use with Latin American immigrants residing in Chile.
\end{abstract}

Keywords: Psychometric properties; threat theory; perceived prejudice; Latin American immigrants; Chile.

\section{Introducción}

La migración es un fenómeno que ha estado presente desde los orígenes de la humanidad, pero es en los últimos 30 años en los que ha adquirido un papel central en la agenda pública (Guillén, Menéndez y Moreira, 2019; Rodicio y Sarceda, 2019; Gutiérrez, et al., 2020). El incremento de inmigrantes a nivel mundial, ha traído consigo una serie de fenómenos sociales en los países de acogida, destacando el prejuicio y discriminación que sufren cotidiniamente estas personas por su origen extranjero.

El impacto de las actitudes prejuiciosas sobre la vida de los inmigrantes ha tomado un rol preponderante en la academia, dado que está catalogado como uno de los principales indicadores que impedirían la integración de estas personas. En esta línea, conocer los niveles de prejuicio percibido es crucial para propiciar políticas de intervención dirigidas a la población inmigrante.
Una forma de medir los niveles de prejuicio percibido, es a través de escalas de actitudes, las cuales se desarrollan considerando el contexto donde está inserta la población objeto de estudio. Actualmente, la Escala de Amenaza Grupal Internalizada (EAGI), es uno de los pocos instrumentos desarrollados para medir el prejuicio percibido por inmigrantes latinoamericanos (BerriosRiquelme, et al., 2018). Este instrumento fue elaborado en España y plantea que los inmigrantes son capaces de internalizar el rechazo y las actitudes negativas que sufren diariamente, porque la población nacional los valora como una amenaza económica y cultural.

Disponer de escalas con adecuadas propiedades psicométricas asegura que los constructos teóricos estén representados en los estudios psicosociales. Cuando se desea utilizar una escala en un contexto distinto al que se elaboró, es necesario evaluar estas propiedades para asegurar que las variables 
tienen el mismo significado y utilidad teórica en la población con la que se trabajará.

Considerando lo anterior, las propiedades psicométricas son el conjunto de indicadores que permiten evaluar la capacidad que tiene una escala para medir el constructo de interés, lo que está dado por: a) Su validez, entendida como el grado en que la evidencia y la teoría soportan las interpretaciones de los puntajes de una prueba e implica acumular evidencias en el contenido, estructura interna y relación con otras variables; y, b) la fiabilidad, la cual consiste en el grado en que una prueba mide con exactitud la auténtica variación de las puntuaciones (American Educational Research Association [AERA], American Psychological Association [APA], y National Council on Measurement in Education [NCME], 2014).

Sobre la base de lo expuesto, el presente estudio tiene como objetivo evaluar las propiedades psicométricas de la Escala de Amenaza Grupal Internalizada (EAGI) en inmigrantes latinoamericanos que viven en Chile. Para esto, se llevaron a cabo dos estudios en dos de las regiones que presentan altos índices de inmigrantes a nivel nacional. En su conjunto, los resultados de ambas investigaciones señalan que la escala posee propiedades psicométricas adecuadas para su uso en el contexto chileno.

Evaluar si la escala funciona en un contexto que posee características similares donde fue creada, es esencial para su incorporación en diversos estudios migratorios que se realicen a lo largo de Chile. Por este motivo, el estudio apunta a contribuir al campo de los estudios migratorios, posibilitando el uso de un instrumento que reúna las características para analizar las variables relacionadas a la vida de los inmigrantes latinoamericanos en su nuevo país.

\section{Inmigración en Chile y el sentimiento de amenaza}

La inmigración latinoamericana en Chile, es un fenómeno que se acentuó durante la última década del siglo XX. En gran parte, el incremento de personas extranjeras encontraría su explicación en la estabilidad política, económica y social, que proyectaba el país dentro del cono Sur (Polo y Serrano, 2018). Sumar a lo anterior, que también esta elección migratoria se vio influenciada por los cierres de frontera que hubo en EEUU y España, dos grandes zonas que históricamente han recibido inmigrantes desde Latinoamérica.

Las últimas cifras oficiales, señalan que los inmigrantes que están en Chile provienen principalmente de Venezuela (455.494), Perú (235.165) y Colombia (161.153), quienes en su conjunto alcanzan a ser $65 \%$ del total de personas extranjeras residentes en el país (Instituto Nacional de Estadística [INE] y Departamento de Extranjería y Migración [DEM], 2020). Respecto a las zonas con mayor presencia de inmigrantes en Chile, la región Metropolitana posee el número más elevado a nivel país (486.568). Por su parte, la región de Tarapacá, con 43.646 personas, tiene el porcentaje $(13,7 \%)$ de inmigrantes más alto en relación a la población nacional (INE, 2018).

La región Metropolitana siempre se ha visto como una zona receptora de inmigrantes nacionales e internacionales, pero ha sido durante los últimos treinta años que ha desarrollado dinámicas económicas clave para el área de servicios, permitiendo ser un polo atractor para personas que poseen una alta y baja calificación (Baeza, 2019). Mientras que, el alto porcentaje de inmigrantes que tiene la región de Tarapacá, encuentra su explicación en que es una zona transfronteriza relacionada a la industria minera, lo que sumado a que es uno de los puertos comerciales más importantes de Chile, la convierten en un asentamiento ideal para aquellas personas que inmigran con fines económicos (Icarte, Torres y Ramos, 2018; Tapia, 2018).

El contexto receptor que tienen los inmigrantes en Chile suele ser hostil, dado que el país no cuenta con una política migratoria integral que favorezca la integración, motivo por el que la población nacional entiende el fenómeno migratorio como un problema social y como una amenaza para la nación (Pavez- 
Berrios-Riquelme, José; Ventura-León, José; Maluenda-Albornoz, Jorge;

Barboza-Palomino, Miguel

Propiedades psicométricas de la escala de amenaza grupal internalizada por inmigrantes

latinoamericanos en Chile

Soto y Colomés, 2018). En este sentido, Stefoni (2011) señala que la ley de extranjería ha construido socialmente al extranjero como una amenaza para los ciudadanos chilenos, puesto que se les aprecia como mano de obra y se les valora según su contribución económica al mercado.

En línea con lo anterior, datos del Instituto de Derechos Humanos (INDH), evidencian que el $68,2 \%$ de la población chilena está de acuerdo con limitar el ingreso de los inmigrantes a Chile, lo que principalmente se explica porque se les asocia como una competencia que les usufructa cupos en el mercado laboral (INDH, 2017). Esta idea es reforzada por los medios de comunicación, quienes desde la década de 1990 posicionan a los inmigrantes como una amenaza para los recursos del país (Correa, 2016).

No es de extrañar, que la población chilena haya desarrollado actitudes negativas hacia los inmigrantes por percibirlos como una amenaza, puesto que el tamaño de la población extranjera es una variable que predice las actitudes negativas hacia este conglomerado (Cea, 2016). Las actitudes negativas que se sustentan desde el enfoque de la amenaza, se caracterizan porque el grupo dominante percibe a los inmigrantes como una competencia ante recursos que suelen ser escasos, como el trabajo o la seguridad social (Berrios-Riquelme, et al., 2018). A la vez, se plantea que el exogrupo posee una cultura distinta y alejada de los valores y de las normas imperantes en la sociedad de acogida (Carmona-Halty y Navas, 2016).

Cuando la inmigración se enmarca como una amenaza asociada a la competencia de recursos económicos y resistencia al cambio cultural, surge un sentimiento de rechazo hacia los extranjeros que es característico de sociedades xenófobas, el cual se operacionaliza a través de prejuicios y actos discriminatorios que los inmigrantes sufren día a día (BerriosRiquelme, et al., 2018).

Diversos estudios dan cuenta cómo los chilenos poseen prejuicios hacia los inmigrantes latinoamericanos por percibirlos como una amenaza. En el norte de Chile,
Carmona-Halty, Navas y Rojas-Paz (2018), encontraron que el sentimiento de amenaza grupal es alto hacia los inmigrantes latinoamericanos y que éste predice el prejuicio afectivo. En el sur de Chile, Mera, et al. (2017), hallaron que el sentimiento de amenaza grupal se asocia con la preferencia de que los inmigrantes se asimilen o se excluyan de la sociedad. Del mismo modo, González, et al. (2017) evidenciaron que los chilenos tienen un alto nivel de percepción de amenaza hacia los extranjeros, donde a menor nivel de escolaridad y menor nivel socioeconómico, mayor será el sentimiento de amenaza hacia este conglomerado.

Desde la perspectiva de los inmigrantes, existe evidencia cualitativa que da cuenta que sienten que son tratados de forma injusta porque son vistos como una amenaza (Silva, Ramírez-Aguilar y Zapata-Sepúlveda, 2018; Gissi, Pinto y Rodríguez, 2019). No obstante, son escasas las aproximaciones cuantitativas que indagan sobre el prejuicio percibido por los inmigrantes y el impacto que tiene en sus vidas, lo que es preocupante, si se considera que las víctimas son capaces de internalizar estas actitudes que repercuten negativamente en las distintas medidas de su salud mental (Urzúa, et al., 2019).

Si bien en Chile es escasa la literatura cuantitativa sobre el prejuicio percibido, en otras latitudes los estudios han encontrado que la autoestima y la identidad étnica son variables protectoras que mitigarían el efecto de las actitudes negativas percibidas. En este sentido, estas emergen como dos de las variables que tendrían una relación inversa con el prejuicio percibido.

En cuanto a la identidad étnica, estaría determinadoporque protegeríaalosinmigrantes de experiencias negativas y estresantes debido al sentimiento de pertenencia grupal (Sellers y Shelton, 2003; Frias-Navarro, et al., 2020). Por este motivo, en el campo de los estudios con inmigrantes latinoamericanos, se sugiere incorporar esta variable cuando se aborda el prejuicio y discriminación (Chavez-Korell y Torres, 2014). Por su parte, la asociación inversa con la autoestima, estaría determinada 
porque es un buen indicador de salud mental que se refleja en el bienestar general (RojasBarahona, Zegers y Förster, 2009) y, por ende, se convierte en un recurso que las personas utilizan para amortiguar sus experiencias negativas (Cast y Burke, 2002).

\section{La Escala de Amenaza Grupal Internalizada}

La Escala de Amenaza Grupal Internalizada (EAGI), es una medida unidimensional que evalúa el grado de consciencia del prejuicio que perciben los inmigrantes debido a su membresía grupal, puesto que la población receptora los trata y los hace sentir que son una amenaza (BerriosRiquelme, et al., 2018). Este sustento, fue considerado para construir en el contexto español este instrumento que evalúa el nivel de prejuicio percibido por inmigrantes desde la perspectiva teórica de la amenaza.

En el estudio de validación de la escala en España, mediante un análisis factorial confirmatorio, se encontraron buenos índices de bondad del ajuste del modelo unifactorial $(\mathrm{CFI}=.970, \mathrm{NFI}=.943$ y RMSEA $=.045)$. También se evidenció una buena consistencia interna $\left(\omega_{\text {total }}=.85\right)$ y correlaciones inversas con el nivel de contacto y las emociones positivas percibidas (Berrios-Riquelme, et al., 2018).

Como señalan Berríos-Riquelme, et al. (2018), la perspectiva teórica de la EAGI plantea que el prejuicio percibido no se genera necesariamente por una interacción directa con la población nacional, también influye el discurso de los partidos políticos y las noticias de la prensa, quienes señalan públicamente a la población inmigrante como una amenaza para el Estado nación. Este contexto, lleva a que los inmigrantes sean conscientes de que son una competencia para los nacionales en el mercado laboral y que su cultura no es apreciada porque no tienen los mismos valores que imperan en el país receptor. De esta manera, la amenaza grupal internalizada es el prejuicio que perciben los inmigrantes en las relaciones asimétricas que sostienen con la población nacional en un contexto hostil.

Son escasos los instrumentos que miden el prejuicio y la discriminación percibida por inmigrantes, pero ninguno de ellos lo realiza desde la perspectiva de la teoría de la amenaza y tampoco se evidencia en la literatura la validación de alguna escala que se enfoque para el trabajo exclusivamente con población de origen latinoamericano. Al igual que en el país donde se realizó el estudio original, en Chile, la realidad de la población inmigrante latinoamericana enfrenta un contexto social, económico y político adverso, así como elevadas tasas de prejuicio y discriminación (Mera, et al., 2017; Carmona, et al., 2018; Pávez-Soto y Colomés, 2018; Urzúa, et al., 2019).

Dado que en Chile no se ha elaborado y tampoco se ha validado un instrumento que permita conocer el prejuicio percibido por inmigrantes, el presente estudio tiene como objetivo evaluar las propiedades psicométricas de la Escala de Amenaza Grupal Internalizada (EAGI) en inmigrantes latinoamericanos en Chile. En específico, se evaluó la evidencia de validez basada en la estructura interna así como en relación con otras variables. Así mismo, se estimó la confiabilidad por consistencia interna. Para cumplir este propósito, se escogió a la población extranjera de origen latinoamericano, dado que es el conglomerado que reporta la mayor discriminación percibida en la última Encuesta de Caracterización Socioeconómica (CASEN) realizada por el Ministerio de Desarrollo Social (2018).

\section{Metodología}

El estudio 1, se realizó en la región de Tarapacá por tener la mayor proporción de personas extranjeras en relación con las nacionales. Se examinó la estructura factorial de la escala mediante un análisis factorial exploratorio (AFE) y se analizó su consistencia interna. El estudio 2, se llevó a cabo en la región Metropolitana porque es la zona que posee el mayor número de inmigrantes a nivel 
Berrios-Riquelme, José; Ventura-León, José; Maluenda-Albornoz, Jorge;

Barboza-Palomino, Miguel

Propiedades psicométricas de la escala de amenaza grupal internalizada por inmigrantes

latinoamericanos en Chile

nacional. En esta segunda etapa, se verificó la estructura interna de la EAGI mediante un análisis factorial confirmatorio (AFC), posteriormente, se examinó su consistencia interna y finalmente, se analizó las evidencias de validez en relación con otras variables. Ambos estudios fueron aprobados por el Comité de Ética de la Universidad de Tarapacá.

\subsection{Estudio 1}

Se realizó durante el segundo semestre del año 2017 en la región de Tarapacá y su propósito fue explorar la estructura factorial y consistencia interna de la EAGI en el contexto chileno.

\section{a. Participantes}

Se encuestó a 378 inmigrantes, pero luego de revisar que los casos tuvieran todos los datos necesarios, la muestra pasó a estar conformada por 320 participantes, donde las tres primeras nacionalidades fueron Bolivia (106), Perú (87) y Colombia (62); las edades fluctuaron entre los 18 y 77 años (Media = 32.53; DE $=8.98$ ). Los criterios de selección fueron: Residir más de un año en Chile y ser mayor de 18 años. El rango de residencia en el país fue desde 1 hasta 56 años (Media = 4.19; $\mathrm{DE}=5.34)$; la percepción de su nivel socioeconómico en su mayoría fue "Media Baja". De estas personas, 136 (43\%) fueron varones y $184(57 \%)$ mujeres, donde 210 $(66 \%)$ reportaron encontrarse en situación regularizada y $110 \quad(34 \%)$ en situación irregular.

\section{b. Procedimiento}

Se realizó un estudio piloto a ocho personas utlizando la técnica de la entrevista cognitiva (Smith-Castro y Molina, 2011) para evaluar el entendimiento y la deseabilidad social de los items, comprobando que estos fueron comprendidos por los inmigrantes y que la redacción no generaba reticencia. En una primera instancia, los participantes fueron seleccionados mediante un muestreo incidental en puntos estratégicos de la ciudad, como supermercados, ferias y peluquerías; posteriormente, se siguió un muestreo por bola de nieve entre las principales nacionalidades de inmigrantes que hay en el país.

A todas las personas se les explicó el objetivo del estudio y la forma en que serían almacenados y analizados sus datos. Posterior a esto, firmaron un consentimiento informado que contenía la descripción del estudio y donde se especificaba que eran libres de dejar de responder el cuestionario en cualquier momento. No se asignaron recompensas de ningún tipo. El cuestionario tuvo la característica de ser autoadministrado y los participantes demoraron entre 25 y 40 minutos en contestar. El investigador responsable, capacitó a dos estudiantes del último año de trabajo social para que lo apoyaran en el proceso de recolección de información.

\section{c. Instrumento}

Siguiendo el propósito de este estudio, se utilizó la Escala de Amenaza Grupal Internalizada [EAGI] de Berrios-Riquelme, et al. (2018). Esta es una escala tipo Likert de seis puntos que evalúa el prejuicio percibido desde la perspectiva de la amenaza, señalando que los inmigrantes sienten que la población nacional los ve como una amenaza para los recursos económicos y para su cultura. Los 10 reactivos que componen la escala pueden ser respondidos de "totalmente en desacuerdo (1)", hasta "totalmente de acuerdo (6)". El puntaje más bajo que puede obtener una persona es de 10 y la puntuación máxima es 60 , siendo el puntaje más alto un indicador de un alto prejuicio percibido por el inmigrante.

La única modificación que tuvieron los ítems fueron las palabras que hacían alusión al país donde se aplica el estudio. Los reactivos de la escala tienen enunciados como 
"Gran parte de los chilenos piensan que los inmigrantes latinoamericanos ocupamos sus puestos de trabajo" o "Los chilenos opinan que los inmigrantes latinoamericanos hablamos y nos expresamos peor que ellos". En la versión original la escala tuvo una consistencia interna de .85 (95\% IC .83 a .87) según el valor alfa de Cronbach y de .85 según el omega de McDonald (1999).

\section{d. Análisis de datos}

El procesamiento de los datos fue con el programa $\mathrm{R}$, versión 3.5.3, utilizando la librería 'psych' (Revelle, 2017). La consistencia interna, se obtuvo mediante una hoja excel programada especialmente para el cálculo de este valor y que ha sido utilizada en la literatura (Ventura-León y CaychoRodríguez, 2017). Se realizó el análisis factorial exploratorio (AFE), previo cálculo de la adecuación muestral, como el coeficiente Kaiser-Meyer-Olkin (KMO $\geq .70)$ y el test de esfericidad de Bartlett $(\mathrm{p}<.05)$, las cuales informan si los datos pueden ser factorizables.

Debido a la naturaleza ordinal de los datos, se utilizó el método de extracción mínimos cuadrados no ponderados con rotación oblimin, que estuvieron basados en correlaciones policóricas (Ledesma, Ferrando y Tosi, 2019). Asimismo, para determinar el número de factores se utilizó el criterio de fijar a uno la cantidad de factores, puesto que es una estrategia que se recomienda cuando se cuenta con una propuesta teórica que avale esta decisión (Lloret, et al., 2017).

\subsection{Estudio 2}

El segundo estudio, se realizó durante el primer semestre del año 2018 en la región Metropolitana y tuvo como objetivo verificar la estructura interna de la EAGI mediante un análisis factorial confirmatorio (AFC), evaluar su fiabilidad y evidenciar la relación con la autoestima e identidad étnica.

\section{a. Participantes}

Inicialmente se contactó a 441 personas, posteriormente, se depuró la base de datos para trabajar con una muestra que contuviera toda la información necesaria. La muestra final estuvo compuesta por 402 inmigrantes latinoamericanos, donde las tres principales nacionalidades fueron Perú (130), Colombia (126) y Venezuela (92). Las edades de los participantes oscilaron de 18 a 65 años (Media $=32.58 ; \mathrm{DE}=8.11)$. En relación a su situación migratoria, $248(62 \%)$ estaban regularizados y $154(38 \%)$ no. Respecto a su situación laboral, $120(30 \%)$ se encontraban sin trabajo, $136(34 \%)$ estaban trabajando con contrato indefinido, 75 (19\%) trabajaban sin contrato y $71(17 \%)$ estaban con contrato a plazo fijo.

\section{b. Procedimiento}

Para recolectar los datos se acudió al Instituto Católico de Migración, lugar en el que se realizó un muestreo incidental durante los meses de enero y julio de 2018. El cuestionario tuvo la característica de ser autoadministrado y los participantes demoraron entre 30 y 45 minutos en completar el documento. El investigador responsable, capacitó a dos trabajadoras sociales con experiencia de trabajo de más de 5 años con población inmigrante para que lo apoyaran durante el trabajo de campo. A los participantes se les garantizó el anonimato en la divulgación de los resultados, así como su participación voluntaria durante todo el proceso. No se entregó ningún tipo de compensación económica para ser parte del estudio.

\section{c. Instrumento}

El instrumento tuvo dos secciones. En el primer apartado del cuestionario, se consultó a los participantes por sus características sociodemográficas: Edad, sexo, situación migratoria, tiempo de residencia en Chile, 
Berrios-Riquelme, José; Ventura-León, José; Maluenda-Albornoz, Jorge;

Barboza-Palomino, Miguel

Propiedades psicométricas de la escala de amenaza grupal internalizada por inmigrantes

latinoamericanos en Chile

nivel de estudios y situación laboral. En el segundo apartado se incluyó la EAGI, la Escala de Autoestima (EA) (Rosenberg, 1965) y la Escala de Identidad Étnica Multigrupo (EIEM) (Roberts, et al., 1999).

1. Escala de Amenaza Grupal Internalizada (EAGI): Esta escala se utilizó considerando los fundamentos teóricos de Berrios-Riquelme, et al. (2018). Asimismo, se utilizó la versión del estudio 1 , donde se reporta una estructura unidimensional y un $\omega$ $=.87$.

2. Escala de Autoestima (EA): De acuerdo con el planteamiento teórico de este manuscrito, el cual señala que el prejuicio percibido tendrá una relación inversa con la autoestima, se utilizó la EA de Rosenberg (1965), en su versión validada para Chile por Rojas-Barahona, et al. (2009). Esta escala cuenta con 10 items que evalúan los sentimientos que una persona tiene a sí misma, donde cinco están en dirección positiva $(1,2,4$, 6 y 7) y cinco están redactados negativamente $(3,5,8,9$ y 10$)$, por los que se les asigna el puntaje inverso para calcular la variable. Las opciones de respuesta son cuatro: Muy en desacuerdo (1), en desacuerdo (2), de acuerdo (3) y muy de acuerdo (4). El puntaje mínimo que se puede alcanzar es 10 y el puntaje máximo es 40 , donde a mayor puntaje, mayor será la autoestima.

\section{Escala de Identidad Étnica} Multigrupo (EIEM): En el apartado teórico de este manuscrito, se planteó que la identidad étnica es una variable protectora del prejuicio percibido, por lo que se espera encontrar una relación inversa entre ambos constructos. Considerando esto, se utilizó la EIEM de Roberts, et al. (1999), en la versión validada al castellano por Smith-Castro (2002). La escala tiene 12 reactivos que miden la identidad étnica, sus puntuaciones van desde "Totalmente en desacuerdo (1)", hasta "Totalmente de acuerdo (4)". Todos los items están en la misma dirección, por lo que al sumar el puntaje, lo mínimo que una persona puede obtener son 12 puntos y lo máximo es 48 puntos. A mayor puntaje, mayor será la identidad étnica que tenga una persona.

\section{d. Análisis de datos}

Considerando los resultados del estudio 1 , con los datos del estudio 2, se realizó un AFC en el entorno del software R versión 3.5.3, utilizando la librería 'lavaan' (Rosseel, et al., 2018). Dada la ordinalidad de los datos, el método de extracción utilizado fue el de mínimos cuadrados no ponderados (ULS) (Brown, 2015). Se utilizaron los siguientes puntos de corte para los índices de bondad de ajuste: Índice de Ajuste Comparativo (CFI $>$.95), Índice de Tucker-Lewis (TLI > .95, $\mathrm{Hu}$ y Bentler, 1999). Valores del RMSEA inferiores a .06 se consideraron como un ajuste satisfactorio, entre .06 y .08 como moderado, y entre .08 y .10 como mediocres (MacCallum, Browne y Sugawara, 1996). Asimismo, el criterio para seleccionar los items al factor fue con un puntaje de corte .40 (Hair, et al., 2017).

Posteriormente, se obtuvo su consistencia interna a través del $\omega$ de McDonald (1999), para lo cual se utilizó una hoja de cálculo en Excel (Ventura-León y Caycho-Rodríguez, 2017). Finalmente, se examinó la evidencia en relación con otras variables, hipotetizando divergencia para la EAGI con las escalas de autoestima y de identidad étnica. Para interpretar el tamaño del efecto se siguieron las recomendaciones de Cohen (1992), donde $\mathrm{r}<.10$ se considera un efecto pequeño, $r=.30$ tamaño del efecto mediano y $r=.50$, un tamaño del efecto grande.

\section{Resultados y discusión}

\subsection{Estudio 1}

\section{a. Análisis Factorial Exploratorio (AFE)}

Se realizó un AFE para estudiar la estructura latente de la escala, donde las medidas de adecuación muestral revelaron la factibilidad de realizar el análisis con la matriz de datos $(\mathrm{KMO}=.84$; Bartlett: $\mathrm{p}<.05)$. De este modo y en concordancia con la teoría, se determinó la extracción de un factor y se 
mantuvieron todos los ítems que tuvieron cargas superiores a .40 (Lloret-Segura, et al., 2014). En la Tabla 1, se aprecia la carga factorial y la comunalidad obtenida por cada item.

\section{Tabla 1}

Matriz Patrón de la Escala de Amenaza Grupal Internalizada

\begin{tabular}{|c|c|c|}
\hline items & F1 & $\mathbf{h}^{2}$ \\
\hline $\begin{array}{l}\text { 1. A los chilenos no les gusta encontrarse con inmigrantes latinoamericanos en sitios públicos } \\
\text { como hospitales, plazas, escuelas, centros comerciales, entre otros. }\end{array}$ & .48 & .23 \\
\hline $\begin{array}{l}\text { 2. Gran parte de los chilenos piensan que los inmigrantes latinoamericanos ocupamos sus } \\
\text { puestos de trabajo }\end{array}$ & .55 & .31 \\
\hline $\begin{array}{l}\text { 3. En Chile se piensa que los inmigrantes latinoamericanos hemos conseguido mayores logros } \\
\text { económicos de los que nos merecemos. } \\
\text { 4. Los chilenos piensan que la mayoría de los inmigrantes latinoamericanos que reciben algún }\end{array}$ & .64 & .41 \\
\hline $\begin{array}{l}\text { tipo de ayuda social o económica, por parte del Estado chileno, podrían vivir sin ella si se } \\
\text { esforzaran más. }\end{array}$ & .70 & .49 \\
\hline $\begin{array}{l}\text { 5. Los chilenos creen que los inmigrantes latinoamericanos estamos presionando demasiado } \\
\text { para que se nos acepte culturalmente en Chile. }\end{array}$ & .73 & .53 \\
\hline $\begin{array}{l}\text { 6. Los chilenos piensan que los inmigrantes latinoamericanos somos honestos, pero aun así } \\
\text { desconfian de nosotros. }\end{array}$ & .61 & .38 \\
\hline $\begin{array}{l}\text { 7. Los chilenos opinan que los inmigrantes latinoamericanos hablamos y nos expresamos } \\
\text { peor que ellos. }\end{array}$ & .60 & .36 \\
\hline $\begin{array}{l}\text { 8. Los chilenos piensan que a los hijos de los inmigrantes latinoamericanos no se les enseña } \\
\text { habilidades y valores adecuados para alcanzar el éxito en Chile. }\end{array}$ & .71 & .51 \\
\hline $\begin{array}{l}\text { 9. Los chilenos piensan que sus creencias y prácticas religiosas son mejores que la de los } \\
\text { inmigrantes latinoamericanos. }\end{array}$ & .69 & .47 \\
\hline $\begin{array}{l}\text { 10. En este país la gente cree que los inmigrantes latinoamericanos tenemos valores distintos } \\
\text { y peores que los chilenos para convivir en la sociedad }\end{array}$ & .69 & .48 \\
\hline Autovalor & & 4.17 \\
\hline$\%$ de varianza & & 42.0 \\
\hline $\begin{array}{l}\omega \\
{[\mathrm{IC} 95 \%]}\end{array}$ & & $\begin{array}{l}.87 \\
{[.85, .89]}\end{array}$ \\
\hline
\end{tabular}

Nota: $\mathrm{h}^{2}$ : Comunalidad; F1: Amenaza Grupal Internalizada; $\omega$ : Coeficiente omega; [IC 95\%]:

representa el intervalo de confianza del coeficiente omega al $95 \%$.

Fuente: Elaboración propia, 2020.

En la Tabla 1, se observa que todas las cargas factoriales son superiores a .30 , siendo la más pequeña el item $1(\lambda=.48)$, lo que subraya la fuerte relación de los items con el constructo evaluado. Este modelo unifactorial es congruente con lo reportado en el estudio original.

\section{b. Consistencia Interna}

La consistencia interna obtenida mediante el coeficiente omega fue buena $\left(\omega_{\text {total }}\right.$ $=.87$, IC $[.85, .89])$, situación que sugiere una buena proporción de varianza común. En este primer estudio, se testeó la estructura factorial 
Berrios-Riquelme, José; Ventura-León, José; Maluenda-Albornoz, Jorge;

Barboza-Palomino, Miguel

Propiedades psicométricas de la escala de amenaza grupal internalizada por inmigrantes

latinoamericanos en Chile

y consistencia interna de la escala. Respecto a los resultados, es posible aseverar que la EAGI posee una estructura unifactorial con elevadas cargas factoriales al igual que el estudio original $\mathrm{y}$, a la vez, presenta una buena consistencia interna. En su conjunto, el primer estudio otorga pruebas de apoyo inicial de la validez y fiabilidad de la escala en el contexto chileno.

\subsection{Estudio 2}

\section{a. Análisis descriptivo de los ítems}

En la Tabla 2, se aprecian los estadísticos descriptivos de los 10 items de la EAGI. El item 6 ostenta el promedio más alto y el item 8 el más bajo. La desviación estándar osciló entre 1.24 a 1.84 . Por otro lado, los valores de asimetría y curtosis se encuentran por debajo a $+/-1.5$, situación que demuestra una aproximación a la distribución multivariada. Finalmente, se presentan las tasas de respuesta para cada una de las alternativas de los items.

Tabla 2

Estadísticos descriptivos de la Escala de Amenaza Grupal Internalizada

\begin{tabular}{|c|c|c|c|c|c|c|c|c|c|c|}
\hline \multirow{2}{*}{ Ítem } & \multirow[t]{2}{*}{ M } & \multirow{2}{*}{$D S$} & \multirow{2}{*}{ g1 } & \multirow{2}{*}{ g2 } & \multicolumn{6}{|c|}{$\%$ de respuesta a cada opción Likert } \\
\hline & & & & & 1 & 2 & 3 & 4 & 5 & 6 \\
\hline 1 & 2.73 & 1.74 & 0.65 & -0.98 & 34 & 23 & 11 & 10 & 11 & 11 \\
\hline 2 & 3.08 & 1.84 & 0.36 & -1.31 & 28 & 19 & 14 & 12 & 11 & 17 \\
\hline 3 & 4.21 & 1.40 & -0.23 & -0.71 & 4 & 4 & 25 & 28 & 12 & 27 \\
\hline 4 & 3.67 & 1.39 & 0.21 & -0.60 & 7 & 8 & 40 & 20 & 10 & 16 \\
\hline 5 & 3.57 & 1.24 & 0.15 & -0.16 & 6 & 10 & 36 & 29 & 10 & 10 \\
\hline 6 & 4.31 & 1.42 & -0.34 & -0.81 & 3 & 7 & 19 & 27 & 14 & 30 \\
\hline 7 & 2.31 & 1.48 & 0.99 & -0.14 & 41 & 26 & 12 & 9 & 9 & 4 \\
\hline 8 & 2.23 & 1.28 & 1.25 & 1.23 & 33 & 35 & 18 & 6 & 3 & 4 \\
\hline 9 & 2.51 & 1.28 & 0.89 & -0.25 & 21 & 40 & 17 & 14 & 5 & 4 \\
\hline 10 & 2.53 & 1.48 & 0.57 & -0.64 & 37 & 14 & 21 & 19 & 4 & 5 \\
\hline
\end{tabular}

Nota: $\mathrm{M}=$ Media, $\mathrm{DS}$ = Desviación estándar; $\mathrm{g} 1$ = Asimetría; $\mathrm{g} 2$ = Curtosis; \% = porcentaje

Fuente: Elaboración propia, 2020. 


\section{b. Análisis Factorial Confirmatorio}

Se modelaron dos estructuras factoriales: En el modelo 1 (M1), se mantuvieron los 10 items de la escala examinando la información teórica y los resultados del AFE del estudio 1 (Boomsma, 2000; Lei y Wu, 2012). El modelo original (M1), no explicó adecuadamente la matriz de covariaciones, por lo que, el instrumento se depuró considerando el mismo criterio de saturación factorial que el estudio $1(\lambda>.40)$. Por ende, se elaboró un modelo 2 (M2), que contiene siete items con cargas factoriales superiores a .50 ; los índices de ajustes comparativos y absolutos, sugieren que el modelo depurado es una buena representación de las relaciones observadas entre cada item y el factor.

En la Tabla 3, se observa el resultado de los dos modelos examinados, evidenciando que el M2 representa mejor los datos de acuerdo con los índices de bondad de ajuste. Asimismo, en la Figura I, es posible observar las cargas factoriales que obtuvo cada modelo.

Tabla 3

Índices de ajuste estadístico y consistencia de la EAGI

\begin{tabular}{|c|c|c|c|c|c|c|c|}
\hline & $\begin{array}{c}\chi^{2} \\
(\mathrm{gl}) *\end{array}$ & $\chi^{2 / \mathrm{gl}}$ & WRMR & CFI & TLI & $\begin{array}{l}\text { RMSEA } \\
\text { [IC 90\%] }\end{array}$ & $\begin{array}{c}\omega \\
{[\text { IC } 95 \%]}\end{array}$ \\
\hline $\begin{array}{c}\text { M1 } \\
\text { (10 ítems) }\end{array}$ & $319.16(35)$ & 9.11 & 1.8 & .92 & .90 & $\begin{array}{c}.14 \\
{[.13, .16]}\end{array}$ & $\begin{array}{c}.79 \\
{[.75, .83]}\end{array}$ \\
\hline $\begin{array}{c}\text { M2 } \\
\text { (7 ítems) }\end{array}$ & 34.39 (14) & 3.13 & .78 & .99 & .99 & $\begin{array}{c}.06 \\
{[.04, .09]}\end{array}$ & $\begin{array}{c}.89 \\
{[.88, .91]}\end{array}$ \\
\hline
\end{tabular}

Fuente: Elaboración propia, 2020.

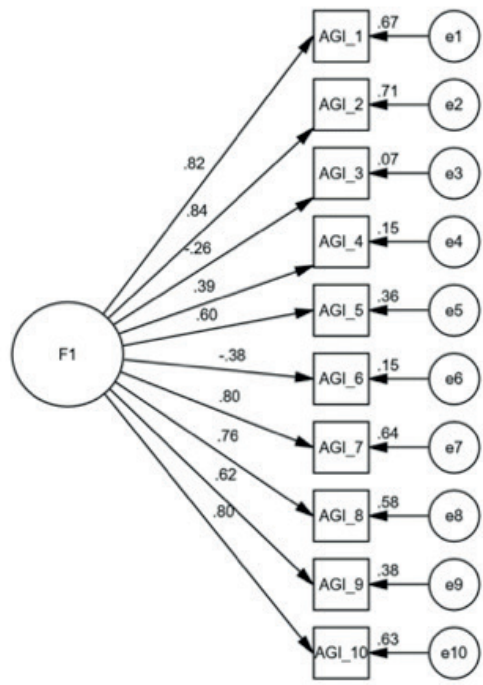

Modelo 1

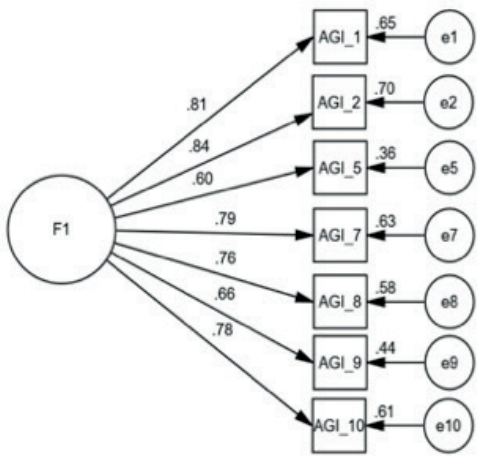

Modelo 2

Fuente: Elaboración propia, 2020.

Figura I: Comparación de ambos modelos factoriales de la Escala de Amenaza Grupal Internalizada 
Berrios-Riquelme, José; Ventura-León, José; Maluenda-Albornoz, Jorge;

Barboza-Palomino, Miguel

Propiedades psicométricas de la escala de amenaza grupal internalizada por inmigrantes

latinoamericanos en Chile

\section{c. Consistencia Interna}

Respecto a la consistencia interna, en el modelo 1 puede ser considerada aceptable $(\omega=$ .79 ; IC $[.75, .83])$ y en el modelo 2 buena $(.89$; IC $[.88, .91])$, tanto en su estimación puntual, como en el límite inferior de su respectivo intervalo de confianza.

\section{d. Evidencias de validez basada en la relación con otras variables}

Para examinar este tipo de validez en la EAGI, se utilizaron las puntuaciones factoriales del Modelo 2, las que se correlacionaron con las puntuaciones de los participantes con la Escala de Autoestima (EA) e Identidad Étnica (EIEM). Los resultados revelan que la AGI se correlaciona de forma moderada e inversa con la autoestima $(\mathrm{r}=-.42$, CFI $=.96$; RMSEA $=.09 ;$ SRMR $=.09)$ y también inversa $\mathrm{y}$ moderadamente con la identidad étnica $(\mathrm{r}=$ $-.41, \mathrm{CFI}=.98 ; \mathrm{RMSEA}=.07$; $\mathrm{SRMR}=.07)$.

Los resultados del segundo estudio, indican que el instrumento presenta buenas evidencias de validez y fiabilidad. Al igual que el estudio original, la escala mostró una estructura unifactorial estable, al mismo tiempo que presentó asociación inversa con la autoestima e identidad étnica, tal como se hipotetizó de acuerdo a la literatura consultada. De esta manera, la EAGI es una medida válida y fiable para medir el prejuicio percibido por inmigrantes latinoamericanos en Chile.

Los hallazgos presentan evidencia empírica para utilizar el constructo con dos variables de importancia en el estudio de las migraciones. Primero, la relación obtenida entre el prejuicio percibido y la autoestima, posee relevancia clínica, puesto que fortalece la conceptualización de que el constructo tiene implicancias negativas en la valoración personal de los inmigrantes. Esta característica sugiere la importancia de continuar investigando en la relación encontrada. Segundo, la asociación inversa hallada entre la EAGI y la identidad étnica, entrega certidumbre sobre la fortaleza teórica del constructo estudiado, puesto que una alta identidad étnica se presenta como un indicador que permite afrontar de mejor manera las actitudes negativas en la sociedad receptora (Cobb, et al., 2019).

La evidencia encontrada proporciona una base inicial para estudiar el prejuicio percibido por inmigrantes latinoamericanos en Chile y se suma a los reportes de la discriminación percibida por este grupo minoritario. Los resultados de esta investigación, contribuyen a confirmar y expandir la literatura sobre la conceptualización de la amenaza grupal internalizada y proveen importantes complementos para su estudio. Un punto a destacar, es que la aplicación de la EAGI se facilitará ahora que tiene 7 items, lo que sumado a su fácil interpretación y utilidad cuando se analizan variables psicosociales relacionadas, la convierten en un instrumento indispensable en futuras investigaciones sobre prejuicio, discriminación y salud mental de inmigrantes en Chile.

\section{Conclusiones}

El prejuicio percibido por los inmigrantes es una realidad creciente en el contexto mundial. Uno de los orígenes de las actitudes negativas hacia esta población, radica en las políticas migratorias que ven al inmigrante como mano de obra y no como una persona que contribuye a la sociedad. Desde esta perspectiva, los inmigrantes son víctimas de prejuicio en la sociedad receptora por ser vistos como una amenaza económica y cultural, lo que impacta negativamente en su salud mental y, a la vez, dificulta su proceso de integración. Considerando esto, el presente artículo tuvo como objetivo evaluar las propiedades psicométricas de la Escala de Amenaza Grupal Internalizada (EAGI) en Chile, para así posibilitar el estudio de este fenómeno y avanzar en propuestas de políticas enfocadas a que los inmigrantes desarrollen estrategias de afrontamiento.

En este contexto, esta investigación aporta evidencia a la comprensión de 
un fenómeno contemporáneo que varias sociedades están viviendo por el aumento de las tasas de inmigrantes en la constante crisis económica que afecta a occidente. En este sentido, los resultados de esta investigación contribuyen al estudio del prejuicio percibido por inmigrantes latinoamericanos.

En este punto, es pertinente mencionar las limitaciones que tuvo el estudio y que deben ser consideradas. Lo primero, es la muestra no probabilística, lo cual limita la validez externa de los hallazgos. Lo segundo, es el tipo de muestreo, que estuvo limitado a ciertas instituciones de la sociedad civil, grupos recreativos y lugares de trabajo donde los investigadores tuvieron acceso. Tercero, esta investigación estuvo enfocada en las cuatro nacionalidades de inmigrantes más numerosas en Chile, por lo que los resultados no podrían ser aplicables a las otras nacionalidades de extranjeros. En cuarto lugar, las muestras obtenidas fueron en el norte y centro de Chile, por lo que puede ser que los inmigrantes que habitan en el sur no presenten las características evidenciadas por los participantes de esta investigación.

Pese a lo anterior, es importante mencionar lo difícil que es encontrar a inmigrantes que quieran participar en este tipo de iniciativas, ya sea porque son poblaciones renuentes a compartir su realidad (Cárdenas y Yáñez, 2012) o porque algunos inmigrantes tienen miedo de que la información que entreguen sea utilizada para ser denunciados en caso de encontrarse en una situación migratoria irregular.

Para futuros estudios, será necesario realizar comparaciones entre nacionalidades de inmigrantes con la finalidad de evaluar cuál grupo necesita mayor atención de las políticas públicas. No obstante, esto requiere un trabajo que continue estudiando las propiedades psicométricas de este instrumento en otras regiones de Chile. Trabajando lo anterior, la escala puede ser utilizada en la evaluación del efecto que tendría como variable mediadora o moderadora en la salud mental de los inmigrantes, proporcionado evidencia que permita desarrollar planes de intervención acordes a la realidad que viven estas personas. A la vez, se recomienda incorporar en esta línea la variable contacto, puesto que la cantidad y la calidad de las relaciones intergrupales también es un predictor del nivel de prejuicio percibido.

A Chile le queda un largo camino por recorrer para consolidar una sociedad libre de prejuicios hacia los inmigrantes, en la que estas personas puedan desenvolverse libremente en los distintos aspectos sociales sin tener miedo al rechazo por ser de otro país y por tener otra cultura. Mientras esto no ocurra, es responsabilidad del mundo académico continuar con la evaluación de medidas que permitan comprender cómo trabajar por el bienestar integral de esta población.

\section{Referencias bibliográficas}

American Educational Research Association - AERA, American Psychological Association - APA, y National Council on Measurement in Education - NCME (2014). Standards for educational and psychological testing. American Educational Research Association. https://www.apa.org/science/ programs/testing/standards

Baeza, P. (2019). Incorporación de inmigrantes sudamericanos en Santiago de Chile: Redes migratorias y movilidad ocupacional. Migraciones Internacionales, 10, 1-28. https://doi. org/10.33679/rmi.v1i1.2145

Berrios-Riquelme, J., Frias-Navarro, D., Pascual-Soler, M., y Badenes-Ribera, L. (2018). Escala de Amenaza Grupal Internalizada por inmigrantes latinoamericanos en España: Evidencias iniciales de fiabilidad y validez. Interciencia, 43(5), 365-371.

Boomsma, A. (2000). Reporting analyses of covariance structures. Structural Equation Modeling, 7(3), 461483. $\quad$ https://doi.org/10.1207/ 
Berrios-Riquelme, José; Ventura-León, José; Maluenda-Albornoz, Jorge;

Barboza-Palomino, Miguel

Propiedades psicométricas de la escala de amenaza grupal internalizada por inmigrantes latinoamericanos en Chile

\section{S15328007SEM0703 6}

Brown, T. A. (2015). Confirmatory factor analysis for applied research. The Guilford Press.

Cárdenas, M., y Yáñez, S. (2012). Nuevas formas de muestreo para minorías y poblaciones ocultas: Muestras por encuestado conducido en una población de inmigrantes sudamericanos. Universitas Psychologica, 11(2), 571578.

Carmona-Halty, M. A., Navas, M., y RojasPaz, P. (2018). Percepción de amenaza exogrupal, contacto intergrupal y prejuicio afectivo hacia colectivos migrantes latinoamericanos en Chile. Interciencia, 43(1), 23-27.

Carmona-Halty, M., y Navas, M. (2016). Análisis psicométrico de la Escala de Percepción de Amenaza Exogrupal (EPAE) en una muestra chilena. Interciencia, 41(11), 788-794.

Cast, A. D., y Burke, P. J. (2002). A theory of self-esteem. Social Forces, 80(3), 1041-1068. https://doi.org/10.1353/ sof.2002.0003

Cea, M. (2016). Immigration as a threat: Explaining the changing pattern of xenophobia in Spain. Journal of International Migration and Integration, 17(2), 569-591. https:// doi.org/10.1007/s12134-015-0415-3

Chavez-Korell, S., y Torres, L. (2014). Perceived stress and depressive symptoms among Latino adults: The moderating role of ethnic identity cluster patterns. The Counseling Psychologist, 42(2), 230-254. https:// doi.org/10.1177/0011000013477905

Cobb, C. L., Meca, A., Branscombe, N. R., Schwartz, S. J., Xie, D., Zea, M. C., Fernandez, C. A., y Sanders, G. L. (2019). Perceived discrimination and well-being among unauthoriz ed Hispanic immigrants: The moderating role of ethnic/racial group identity centrality. Cultural Diversity and Ethnic Minority Psychology, 25(2), 280-287. $\quad$ https://doi.org/10.1037/ $\underline{\mathrm{cdp} 0000227}$

Cohen, J. (1992). A power primer. Psychological Bulletin, 112(1), 155 159. https://doi.org/10.1037/0033$\underline{2909.112 .1 .155}$

Correa, J. (2016). La inmigración como "problema" o el resurgir de la raza. Racismo general, racismo cotidiano y su papel en la conformación de la Nación. En. M. E. Tijoux (Ed.), Racismo en Chile: La piel como marca de inmigración (pp. 35-46). Editorial Universitaria.

Frias-Navarro, D., Botvyn, Y., Pascual-Soler, M., y Berrios-Riquelme, J. (2020). Multigroup ethnic identity measure: Factorial structure in Ukrainian immigrants in western Europe. Opción, 36(92), 914-938.

Gissi, E. N., Pinto, C., y Rodríguez, F. (2019). Inmigración reciente de colombianos y colombianas en Chile. Sociedades plurales, imaginarios sociales y estereotipos. Estudios Atacameños, (62), 127-141. https://dx.doi. org/10.22199/issn.0718-1043-2019$\underline{0011}$

González, R., Bargsted, M., Miranda, D., Carvacho, H., De Tezanos, P., y Doña-Reveco, C., Tropp, L., van Laar, C., Álvarez, B., Valdenegro, D., Volgger, A., y Cheyre, M. (2017). Módulo 1: Cohesión social y migración. Resultados primera ola. Estudio Longitudinal de Chile - ELSOC. (Notas COES de Política Pública No. 1). COES. https://coes. cl/publicaciones/n1-elsoc-cohesionsocial-y-migracion-2/

Guillén, J. C., Menéndez, F. G., y Moreira, T. K. (2019). Migración: Como fenómeno social vulnerable y salvaguarda de 
los derechos humanos. Revista de Ciencias Sociales (Ve), XXV(E-1), 281-294. https://doi.org/10.31876/rcs. v25i1.29619

Gutiérrez, J. M., Romero, J., Arias, S. R., y Briones, X. F. (2020). Migración: Contexto, impacto y desafío. Una reflexión teórica. Revista de Ciencias Sociales (Ve), XXVI(2), 229313. https://doi.org/10.31876/rcs. v26i2.32443

Hair, J. F., Hult, G. T. M., Ringle, C., y Sarstedt, M. (2017). A primer on Partial Least Squares Structural Equation Modeling (PLS-SEM). Sage Publishing.

Hu, L., y Bentler, P. M. (1999). Cutoff criteria for fit indexes in covariance structure analysis: Conventional criteria versus new alternatives. Structural Equation Modeling, 6(1), 1-55. https://doi. org/10.1080/10705519909540118

Icarte, L., Torres, N., y Ramos, R. (2018). La Zona Franca de Iquique y su impacto en el trabajo informal de mujeres migrantes fronterizas. Polis, (51), 6387. https://dx.doi.org/10.32735/s0718$\underline{6568 / 2018-n 51-1350}$

Instituto Nacional de Derechos Humanos INDH (2017). Informa Anual 2017. Situación de los Derechos Humanos en Chile 2017. INDH. https://www. indh.cl/destacados/informe-anual/

Instituto Nacional de Estadísticas - INE (2018). Características de la inmigración internacional en Chile, Censo 2017. INE. http://www.censo2017.cl/ descargas/inmigracion/181123documento-migracion.pdf

Instituto Nacional de Estadísticas - INE y Departamento de Extranjería y Migración - DEM (2020). Estimación de personas extranjeras residentes habituales en Chile al 31 de diciembre 2019. https://www.ine.cl/ docs/default-source/demografia-y- migracion/publicaciones-y-anuarios/ migraci $\% \mathrm{C} 3 \% \mathrm{~B} 3 \mathrm{n}$-internacional/ estimaci $\%$ C3\%B3n-poblaci $\% C 3 \%$ B3n-extranjeraen-chile-2018/estimaci $\%$ C3\%B3npoblaci $\%$ C 3\%B3n-extranjera-enchile-2019-metodolog\%C3\%ADa. pdf?sfvrsn $=5$ b $145256 \quad 6$

Ledesma, R. D., Ferrando, P. J., y Tosi, J. D. (2019). Uso del Análisis Factorial Exploratorio en RIDEP. Recomendaciones para autores y revisores. Revista Iberoamericana de Diagnóstico y Evaluación - e Avaliação Psicológica. RIDEP, 52(3), 173-180. $\quad \underline{\text { https://doi.org/10.21865/ }}$ $\underline{\text { RIDEP52.3.13 }}$

Lei, P-W., y Wu, Q. (2012). Estimation in structural equation modeling. In Hoyle, R. H. (Ed.), Handbook of structural equation modeling (pp. 164179). Guildford Press.

Lloret, S., Ferreres, A., Hernández, A., y Tomás, I. (2017). El análisis factorial exploratorio de los ítems: Análisis guiado según los datos empíricos y el software. Anales de Psicología, 33(2), 417-432. https://doi.org/10.6018/ analesps.33.2.270211

Lloret-Segura, S., Ferreres-Traver, A., Hernández-Baeza, A., y TomásMarco, I. (2014). El análisis factorial exploratorio de los ítems: Una guía práctica, revisada y actualizada. Anales de Psicología, 30(3), 1151$1169 . \quad$ http://dx.doi.org/10.6018/ analesps.30.3.199361

MacCallum, R. C., Browne, M. W., y Sugawara, H. M. (1996). Power analysis and determination of sample size for covariance structure modeling. Psychological Methods, 1(2), 130149. https://doi.org/10.1037/1082989X.1.2.130

McDonald, R. P. (1999). Test theory: $A$ unified treatment. Taylor \& Francis Group. https://doi. 
Berrios-Riquelme, José; Ventura-León, José; Maluenda-Albornoz, Jorge;

Barboza-Palomino, Miguel

Propiedades psicométricas de la escala de amenaza grupal internalizada por inmigrantes

latinoamericanos en Chile

org/10.4324/9781410601087

Mera, M. J., Martínez-Zelaya, G., Bilbao, M. A., y Garrido, A. (2017). Chilenos ante la inmigración: Un estudio de las relaciones entre orientaciones de aculturación, percepción de amenaza y bienestar social en el Gran Concepción. Universitas Psychologica, 16(5), 1-14. https://doi.org/10.11144/Javeriana. upsy16-5.cier

Ministerio de Desarrollo Social MDS (2018). Encuesta de Caracterización Socieconómica 2017. MDS. http://observatorio. ministeriodesarrollosocial.gob.cl/ casen-multidimensional/casen/docs/ Resultados Inmigrantes casen 2017. pdf

Pávez-Soto, I. y Colomés, S. (2018). Derechos humanos y política migratoria. Discriminación arbitraria en el control de fronteras en Chile. Polis, 17(51), 113-136.

Polo, S., y Serrano, E. (2018). Nueva república, nuevo horizonte, nuevo porvenir: Las migraciones colombianas hacia Chile, 1990-2016. Novum Jus, 12(1), 165-188. https://doi.org/10.14718/ NovumJus.2017.12.1.7

Revelle, W. (2017). Package 'psych'. The Comprehensive $R$ Archive Network. https://cran.r-project.org/web/ packages/psych/psych.pdf.

Roberts, R. E., Phinney, J. S., Masse, L. C., Chen, Y. R., Roberts, C. R., y Romero, A. (1999). The structure of ethnic identity in young adolescents from diverse ethnocultural groups. Journal of Early Adolescence, 19(3), 301-322. https://doi.org/10.1177/02724316990 19003001

Rodicio, M. L., y Sarceda, M. C. (2019). Inserción sociolaboral de mujeres emigrantes retornadas: Desde Venezuela a España. Revista de
Ciencias Sociales (Ve), XXV(4), 11-21.

Rojas-Barahona, C. A., Zegers, B., y Förster, C. E. (2009). La escala de autoestima de Rosenberg: Validación para Chile en una muestra de jóvenes adultos, adultos y adultos mayores. Revista Médica de Chile, 137(6), 791-800. http://dx.doi.org/10.4067/S0034$\underline{98872009000600009}$

Rosenberg, M, (1965). Society and the adolescent self-image. Princeton University Press.

Rosseel, Y., Obersky, D., Byrnes, J., Vanbrabant, L., Savalei, V., Merkle, E., y Chow, M. (2018). Package "lavaan" 0.6.2. https://cran.rproject. org/web/packages/lavaan/lavaan.pdf

Sellers, R. M., y Shelton, J. N. (2003). The role of racial identity in perceived racial discrimination. Journal of Personality and Social Psychology, 84(5), 10791092. https://doi.org/10.1037/0022$\underline{3514.84 .5 .1079}$

Silva, J., Ramírez-Aguilar, F., y ZapataSepúlveda, P. (2018). Experiencias laborales de mujeres migrantes afrocolombianas en el norte de Chile. Interciencia, 43(8), 544-551.

Smith-Castro, V. (2002). La Escala de Identidad Étnica Multigrupo (EIEM) en el contexto costarricense. Actualidades en Psicología, 18(105), 47-67.

Smith-Castro, V., y Molina, M. (2011). Cuaderno metodológico 5. La entrevista cognitiva: Guía para su aplicación en la evaluación y mejoramiento de instrumentos de papel $y$ lápiz. Instituto de Investigaciones Psicológicas, Universidad de Costa Rica. http://iip.ucr.ac.cr/sites/default/ files/contenido/Entrevista $\% 20$ Cognitiva\%20\%282011\%29.pdf

Stefoni, C. (2011). Ley y política migratoria en Chile. La ambivalencia en la comprensión del migrante. En B. 
Feldman-Bianco, L. Rivera, C. Stefoni y M. Villa (Eds.), La construcción social del sujeto migrante en América Latina: prácticas representaciones y categorías (pp. 79-109). FLACSOEcuador.

Tapia, M. (2018). Prácticas sociales fronterizas entre Chile y Bolivia, Movilidad, Circulación y Migración. Siglos XX y XXI. Intus-Legere Historia, 12(1), 66-86.

Urzúa, A., Cabrera, C., Calderón, C., y Caqueo-
Urízar, A. (2019). The mediating role of sel-esteem on the relationship between perceived discrimination and mental health in South American immigrants in Chile. Psychiatry Research, 271, 187-194. https://doi. org/10.1016/j.psychres.2018.11.028

Ventura-León, J. L., y Caycho-Rodríguez, T. (2017). El coeficiente Omega: un método alternativo para la estimación de la confiabilidad. Revista Latinoamericana de Ciencias Sociales, Niñez y Juventud, 15(1), 625-627. 